\title{
KVT ist der Lichttherapie langfristig überlegen
}

Fragestellung: Sind kognitive Verhaltenstherapie (KVT) und Lichttherapie bei saisonalen affektiven Störungen langfristig vergleichbar wirksam?

Hintergrund: Lichttherapie gilt als hoch wirksame Methode in der Akutbehandlung saisonaler affektiver Störungen (SAD). Wegen der oft niedrigen Langzeitcompliance zu deren täglicher Anwendung besteht allerdings ein Rezidivrisiko. Die speziell auf saisonale affektive Störungen zugeschnittene KVT stellt eine alternative und auf längere Sicht möglicherweise praktikablere Therapieform dar. In Pilotstudien zeigten beide Therapien in der Akutbehandlung vergleichbare Effekte, einen Winter später erwies sich die KVT gegenüber der Lichttherapie als überlegen.

Patienten und Methodik: Im Rahmen einer randomisierten, prospektiven Studie wurden 177 Patienten mit rezidivierender Major Depression (nach DSM-IV-TR) mit saisonal abhängigem Verlauf entweder der KVT $(n=88)$ oder der Lichttherapie $(n=89)$ zugeteilt. Der Behandlungszeitraum umfasste sechs Wochen. Die Lichttherapie bestand aus der

Rohan KJ, Meyerhoff J, Ho SY et al. Outcomes one and two winters following cognitive-behavioral therapy or light therapy for seasonal affective disorder. Am J Psychiatry 2016; 173: 244-51 täglichen Exposition mit hellem künstlichen Licht (Stärke 10.000 Lux) unmittelbar nach dem Erwachen, für je $15 \mathrm{Mi}$ nuten bis maximal zwei Stunden. Patienten wurden zur kontinuierlichen Fortsetzung der täglichen Lichttherapie bis zur Spontanremission geraten. Die KVT erstreckte sich über zwölf 90-minütige Gruppensitzungen ( 2 x pro Woche). Im ersten Folgeherbst nach Behandlungsende wurde der Depressionsstatus telefonisch erfasst, in und zwei Winter später fanden persönliche Follow-up-Erhebungen statt. Das wichtigste Outcome-Maß war der Rezidivstatus im strukturierten Interviewleitfaden zur Hamilton Depression Rating Skala - Version für SAD (SIGH-SAD), ein Rezidiv lag vor wenn der Gesamtscore 20 oder mehr Punkte betrug. Weitere OutcomeMaße waren der Schweregrad im SIGH-SAD und im BDI-II sowie der Remissionsstatus im BDI-II (Summenscore $<8$ Punkte).

Ergebnisse: Zum Follow-up-Zeitpunkt 1 einen Winter nach der sechswöchigen Behandlung bestand bezüglicher aller Outcomes (Rezidiv- bzw. Remissionsstatus und Schweregrad) kein signifikanter Unterschied zwischen der KVT- und der LichttherapieGruppe. Im zweiten Winter erwies sich die KVT gegenüber der Lichttherapie als überlegen: die Rezidivrate unter den KVT-Patienten war niedriger (27,3\% vs. $45,6 \%$ ), der Schweregrad geringer und der Anteil an Remissionen höher (68,3\% vs. 44,5\%). Zudem bestand in der KVT-Bedingung ein stärkerer Zusammenhang zwischen dem Ausbleiben eines Rezidivs im ersten und zweiten Winter (relatives Risiko: 5,12\% vs. 1,92\%).

Schlussfolgerungen: Lichttherapie und KVT sind bei akuten SAD vergleichbar wirksam. Die KVT erzielt jedoch länger andauernde Effekte und eignet sich zur Rezidivprävention.

\section{- Kommentar von Katrin Frey, Erlangen}

\section{Gute Evidenz für die Wirksamkeit von Lichttherapie und KVT bei SAD}

Die Autoren dieser Studie nutzten erstmals ein prospektives Design, um die Langzeitwirksamkeit von KVT und Lichttherapie im direkten Vergleich zu untersuchen und somit dem wiederkehrenden Verlaufsmuster des zugrundeliegenden Störungsbildes gerecht zu werden. Mit einem JADAD-Score von vier Punkten erfüllt die Studie hohe methodische Standards. Anbetracht der hohen Prävalenz von SAD sowie der weiten Verbreitung von KVT und Lichttherapie als gängige leitliniengetreue Behandlungsmethoden ist die Studie von hoher klinischer Relevanz. Zudem wurden Patienten mit komorbiden Störungen nicht ausgeschlossen, sodass eine hohe ökologische Validität vorliegt. Das Hauptergebnis bestätigt erwartungsgetreu eine längerfristige Überlegenheit der KVT gegenüber der Lichttherapie, in der Akutbehandlung bis zu einem Jahr danach ist die Wirksamkeit der Studie zufolge vergleichbar. Als Begründung wird die Praktikabilität angeführt, die Lichttherapie erfordert die fortsetzende tägliche Anwendung mit Symptombeginn im Herbst/Winter (Dauer: etwa 30 Minuten), die KVT dagegen vermittelt flexibler einsetzbare und alltagskompatiblere Strategien. Es besteht jedoch weiterer Forschungsbedarf, da in beiden Bedingungen nach sechswöchi- ger Behandlung etwa ein Viertel der KVT- und etwa die Hälfte der Lichttherapie-Patienten weiter behandlungsbedürftig waren. Zudem sind die Daten zur Identifikation der Patientengruppe, die von einer Kombinationsbehandlung einen additiven Effekt erzielen können, bisher noch unzureichend. Zusammenfassend stellt die Lichttherapie ein kostengünstiges und in der Akutbehandlung hoch wirksames Behandlungsinstrument dar, das zur kurzfristigen Linderung depressiver Symptome und möglicherweise zur Überbrückung der Wartezeit auf einen Therapieplatz (KVT) sehr geeignet ist.

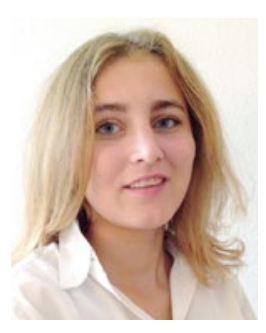

Katrin Frey, M.Sc., Erlangen

Psychologin, Psychiatrische und

Psychotherapeutische Klinik,

Universitätsklinikum Erlangen

E-Mail: Katrin.Frey@uk-erlangen.de 